\title{
有机叠氮化合物的合成研究进展
}

\author{
江玉波 ${ }^{*}, a$ 匡春香 ${ }^{*, b} \quad$ 韩春美 $^{c}$ 王 红 ${ }^{a}$ 梁雪秋 $^{a}$ \\ ( ${ }^{a}$ 昆明理工大学理学院 昆明 650500) \\ ( ${ }^{b}$ 同济大学化学系 上海 200092) \\ ( ${ }^{c}$ 昆明理工大学机电工程学院 昆明 650500)
}

\begin{abstract}
摘要 综述了有机叠氮化合物近些年的合成方法研究进展. 分别按不同类别进行了阐述，包括芳基叠氮、烯基叠氮、 烷基叠氮、酰基叠氮等, 并对部分反应机理进行了讨论.

关键词 有机叠氮; 合成; 进展

\section{Advances in the Synthesis of Organic Azides}

\author{
Jiang, Yubo ${ }^{*, a}$ Kuang, Chunxiang ${ }^{*, b} \quad{\text { Han, } \text { Chunmei }^{c} \quad \text { Wang, Hong }^{a} \quad \text { Liang, Xueqiu }}^{a}$ \\ ( ${ }^{a}$ Faculty of Science, Kunming University of Science and Technology, Kunming 650500) \\ ( ${ }^{a}$ Department of Chemistry, Tongji University, Shanghai 200092) \\ ( ${ }^{c}$ School of Mechanical and Electronic Engineering, Kunming University of Science and Technology, Kunming 650500)
}

\begin{abstract}
The recent advances in the synthesis of organic azides are reviewed, based on the categories of these compounds including alkyl azides, alkenyl azides, aryl azides and acyl azides. Mechanism of some reactions is also discussed.

Keywords organic azides; synthesis; advances
\end{abstract}

有机叠氮化合物含有叠氮基高能活性官能团，是一 类重要的有机合成中间体，已广泛应用于有机合成 ${ }^{[1]}$ 、 化学生物学 ${ }^{[2]}$ 、功能材料 ${ }^{[3]}$ 及临床医药(Scheme 1)等领 域.

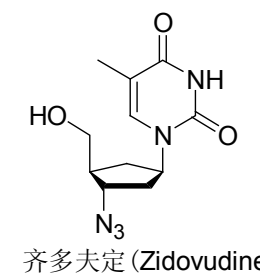
(抗艾滋病药)

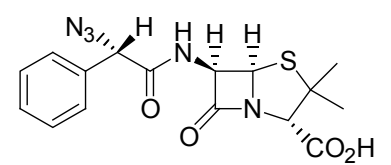

阿度西林 (Azidocillin) (抗菌药)
Scheme 1

第一例有机叠氮化合物是由德国化学家 Griess $^{[4]}$ 于 1864 年在实验室中制备获得, 他使用氨与过澳化重氮 苯反应得了苯基叠氮. 叠氮化合物具有一定的毒性及潜 在爆炸性，在第一次世界大战期间曾代替雷永作为炸药 引爆剂, 因而当时学者对叠氮化物产生了畏惧心理并敬
而远之, 该类化合物曾一度无人研究.

近期，随着人们对叠氮化物性质的全面认识及其实 验安全技术的提高, 该类化合物重新获得研究者的重 视, 特别是本世纪初 Sharpless 等 ${ }^{[]}$将叠氮化合物与端炔 的 1,3-偶极环加成发展成 “点击” 反应以来, 有机叠氮 化合物在合成 1,2,3-三氮唑类化合物等众多相关领域的 应用发展尤为迅速 ${ }^{[6]}$, 这对该类化合物的种类及合成方 法提出了更高要求.

有机叠氮化合物主要是通过含不饱和键及某些极 性键的底物与能提供叠氮基的前体发生加成或亲核取 代反应获得, 常见能提供叠氮基的前体有叠氮酸 $\left(\mathrm{HN}_{3}\right)$ 、 叠氮化钠 $\left(\mathrm{NaN}_{3}\right)$ 、三甲硅基叠氮(TMSA)、二苯基磷酰 基叠氮(DPPA)、三丁基锡基叠氮(TBSnA)、叠氮乙酸乙 酯(AAE)、叠氮化四丁基铵(TBAA)等, 其中最常用的是 叠氮化钠. 本综述主要阐述了近些年有机叠氮化合物合 成的发展, 并对这些方法进行了分析及展望.

\footnotetext{
*E-mail: ybjiang@kmust.edu.cn

Received June 24, 2012; revised July 18, 2012; published online July 28, 2012.

Project supported by the National Natural Science Foundation of China (No. 21262020) and the Science and Technology Planning Project of Yunnan Province (No. KKSY201207047).

国家自然科学基金(No. 21262020)和云南省科技厅(No. KKSY201207047)资助项目.
} 


\section{1 芳基叠氮的合成}

\section{1 芳胺重氮化反应}

利用芳胺重氮化反应制备芳基叠氮化合物是该类 化合物合成的经典方法，反应常分两步进行(Eq. 1), 第 一步是芳氨 1 氧化得到重氮盐, 常以亚硝酸钠作为氧化 剂, 在强酸性环境中进行; 第二步是重氮盐的分解并进 一步叠氮化得到目标产物 2.

$$
\underset{1}{\mathrm{ArNH}_{2}} \stackrel{\text { 1) } \mathrm{NaNO}_{2}, \mathrm{H}_{2} \mathrm{O}, \mathrm{HX}, 0 \sim 5{ }^{\circ} \mathrm{C}}{\stackrel{2}{\longrightarrow} \mathrm{NaN}_{3}, 0 \sim 5{ }^{\circ} \mathrm{C} \text {; r.t. }} \underset{2}{\mathrm{ArN}_{3}}
$$

此方法具有成本低廉、反应高效等优点, 至今仍被 广泛使用 ${ }^{[7]}$, 其缺点是需要在强酸性环境下进行, 有时 会影响底物结构及官能团的兼容性. Zarei 等 ${ }^{[8]}$ 使用硅载 硫酸 3 作为酸化试剂, 芳胺在室温条件下即可转化为相 应叠氮化合物(Scheme 2).

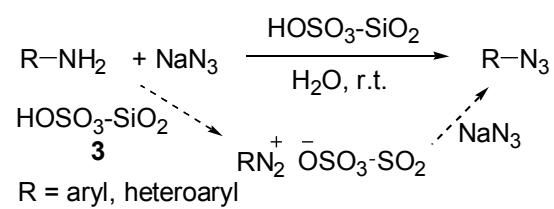

Scheme 2

该方法所生成的重氮盐中间体比较稳定，在室温下 可以保存数日, 操作比一般无机酸参与的反应更为简 便.

\section{2 缺电芳卤直接亲核取代反应}

缺电子芳环的芳卤键被活化, 其亲电性增强, 易发 生多种亲核取代反应. Wilson 等 ${ }^{[9]}$ 使用含活化碳卤键的 2-氯吡啶系类化合物 4 和叠氮化钠直接发生亲核取代反 应而得到芳基叠氮类化合物 $\mathbf{5}$, 所含取代基为吸电子基 子时更有利于反应的进行. 该反应产物实际上是叠氮化 合物 5 与四氮唑 6 的平衡混合物(Scheme 3).
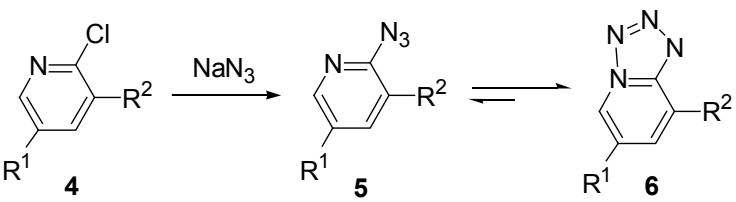

$\mathrm{R}^{1}=\mathrm{R}^{2}=\mathrm{H} ; \mathrm{R}^{1}=\mathrm{NO}_{2}, \mathrm{R}^{2}=\mathrm{H} ; \mathrm{R}^{1}=\mathrm{H}, \mathrm{R}^{2}=\mathrm{NO}_{2} ; \mathrm{R}^{1}=\mathrm{R}^{2}=\mathrm{NO}_{2}$

Scheme 3

\section{3 卤代烃催化偶联}

$\mathrm{Ma}$ 等 $^{[10]}$ 通过卤代烃与叠氮钠的催化偶联反应获得 了有机叠氮化合物, 他们以碘化亚铜 $/ L$-脯氨酸为催化 体系在较温和条件下实现了碳-氮偶联(Scheme 4).

$$
\begin{aligned}
& \text { Cul (5 } 10 \mathrm{~mol} \%) \text { ), L-proline (10 } 20 \mathrm{~mol} \%) \\
& \mathrm{Ar}-\mathrm{I}+\mathrm{NaN}_{3} \stackrel{\mathrm{NaOH}(10 \sim 20 \mathrm{~mol} \%)}{\mathrm{DMSO}, 60^{\circ} \mathrm{C}} \mathrm{Ar}-\mathrm{N}_{3} \\
& \text { Cul (10 mol\%), L-proline (30 mol\%) } \\
& \underset{8}{\mathrm{Ar}-\mathrm{Br}}+\mathrm{NaN}_{3} \frac{\mathrm{NaOH}(30 \mathrm{~mol} \%)}{\mathrm{EtOH} / \mathrm{H}_{2} \mathrm{O}(\mathrm{V}: V=7: 3), 95^{\circ} \mathrm{C}} \mathrm{Ar}-\mathrm{N}_{3} \\
& \underset{9}{2}+\mathrm{NaN}_{3} \frac{\mathrm{Cul}(10 \mathrm{~mol} \%), \mathrm{L} \text {-proline sodium }(20 \mathrm{~mol} \%)}{\mathrm{EtOH} / \mathrm{H}_{2} \mathrm{O}(\mathrm{V}: \mathrm{V}=7: 3), 95^{\circ} \mathrm{C}} \mathrm{R} \\
& \mathrm{R}=\text { aryl or alkyl }
\end{aligned}
$$

\section{Scheme 4}

芳基碘代物 7 和芳基溴代物 8 在该催化体系下均能 顺利发生偶联反应得到目标化合物，只是条件略有不 同，但是烯基卤化物中只有碘代物 9 能进行偶联反应得 到相应产物.

\section{4 有机硼酸催化偶联}

Guo 等 ${ }^{[11]}$ 发现芳基硼酸 10 和叠氮钠在硫酸铜催化 下即能顺利反应，生成芳基叠氮化合物. 此反应条件温 和，在室温下即可进行，多种官能团不受影响，烯基砋 酸 11 也能高效的转化为烯基叠氮, 且立体选择性保持 (Scheme 5).

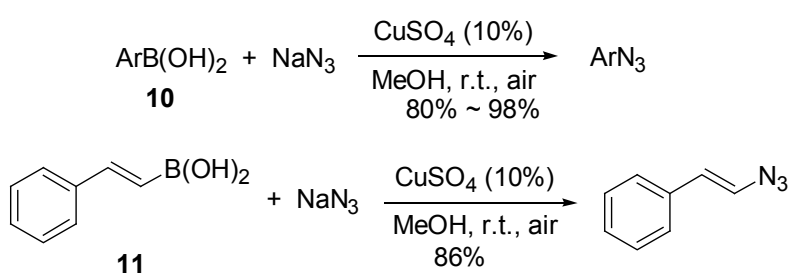

Scheme 5

该方法的另一特点是反应条件要求不苛刻. “铜源” 可取范围广泛，除了硫酸铜外还可以是醋酸铜、碘化亚 铜、氯化亚铜等; 溶剂除了甲醇外，乙醇、甲醇/水也可 以，甚至在水中反应亦能获得中等收率的产物. 此方法 可用于原位生成叠氮化物并进一步直接参与 $1,2,3$-三氮 唑的合成，从而省去叠氮化物的制备及分离过程.

\section{5 芳基叠氮直接衍生化}

2011 年 Kuang 等 ${ }^{[12]}$ 报道了芳基叠氮的直接溴化反 应，他们以三氯化铁为催化剂、以 $N$-溴代丁二酰亚胺 (NBS)作为溴化试剂，在较温和条件下对芳基叠氮化合 物 12 直接进行衍生, 获得新的含卤叠氮化合物 13 (Eq. 2).

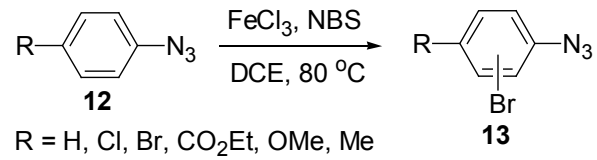


本反应条件主要适用于对位取代的芳基叠氮, 溴化 反应位置遵循取代基的定位效应及其规律, 溴通常取代 叠氮基邻位的氢; 若取代基供电性强于叠氮基团, 溴原 子则更容易取代该基团的邻位氢, 此方法还适用于 2-叠 氮基噻吩及 1 -叠氮基萗的溴化衍生.

\section{2 烯基叠氮的合成}

烯基叠氮化合物可通过烯基卤及烯基硼酸的催化 偶联反应合成(Schemes 4 5), 还可由下列方法获得.

\section{1 肉桂酸及肉桂酸酯的加成/消除反应}

Nair 等 ${ }^{[13]}$ 发现, 在硝酸铈铵(CAN) 作用下, 肉桂酸 酯及 $\alpha, \beta$-不饱和酮 14 可与叠氮钠反应分别生成相应 $\alpha-$ 叠氮基肉桂酸酯及 $\alpha$-叠氮基- $\alpha, \beta$-不饱和酮 16 (Scheme 6).

反应时叠氮离子在硝酸铈铵作用下首先与化合物 14 发生加成反应, 形成中间体 15 , 接着再发生消除过 程, 脱去一分子硝酸得到产物烯基叠氮 16, 其立体构型 保持. 若底物为肉桂酸衍生物 17 , 则生成 $\beta$-叠氮基苯乙 烯衍生物 18 (Scheme 7).

在 Scheme 7 的反应中, 肉桂酸 17 先在硝酸铈铵及 叠氮钠作用下生成 19 , 后者消除硝基同时发生脱羧反 应即得到 $\beta$-叠氮基苯乙烯衍生物 18 , 其缺点是没有立 体选择性, 得到是 $E$ 式及 $Z$ 式等比例混合物.

\section{2 烯烃的加成/消除反应}

Hassner 等 ${ }^{[14]}$ 利用叠氮化碘 $\left(\mathrm{IN}_{3}\right)$ 和烯烃合成了烯基 叠氮化合物. 反应中烯烃 20 先和叠氮化碘发生反式加 成得到中间体 21, 后者进一步发生消除脱去一份子碘
化氢得到烯基叠氮 22. 该反应适合于对称链烯烃或环 烯烃, 而不对称烯烃则得到的是无区域选择性的混合产 物(Scheme 8).

\section{3 醛的 Knoevenagel 反应}

利用芳香醛和 $\alpha$-叠氮基乙酸甲酯在碱的作用下发 生 Knoevenagel 缩合可以获得相应烯基叠氮, 这种方法 至今应用仍为广泛, Seeberger 等 ${ }^{[15]}$ 报道了利用叠氮基丙 烯酸酯在连续流动反应器中通过热分解制备吲哚及相 关杂环化合物的方法, 他们所用的叠氮类化合物 $\mathbf{2 3}$ 就 是通过 Knoevenagel 缩合法获得的(Eq. 3). 该方法对于 杂环芳香醛同样适用.

\section{4 烯基碘盐取代}

利用烯基苯基碘的四氟硼酸盐 24 与叠氮钠反应可 以获得烯基叠氮类化合物 $\mathbf{2 5}$, 反应在室温下经 $0.5 \mathrm{~h}$ 即 可完成, 且立体结构保持(Eq. 4) ${ }^{[16]}$.

此反应条件温和, 收率高, 若使用硫氧酸钾代替叠 氮化钠参与反应则可得到烯基硫氰化物, 不过其立体选 择性不佳, 常得到顺反异构体混合产物.

\section{5 环氧丙烷衍生物开环消除}

Chakraborty 等 ${ }^{[17]}$ 发现, 以 DMF 为溶剂, 含有三甲 基硅基的环氧丙烷类化合物 26 与叠氮钠反应可立体选 择性生成烯基叠氮 27 (Scheme 9).

反应中环氧丙环开环后得到中间体构象 $\mathbf{2 8}$, 后者 旋转后得到氧原子与三甲基硅基同侧的构象 29 , 再经 消除三甲基硅氧负离子即得到产物 27, 产物构型由氧 原子与三甲硅基同侧消除的立体要求决定.

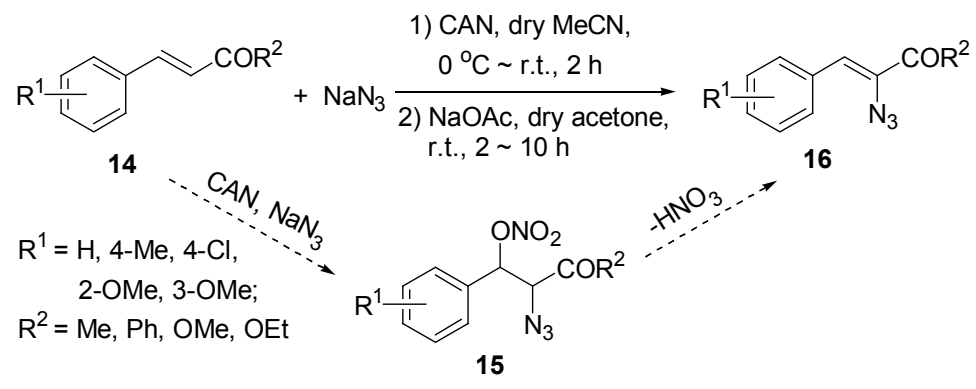

Scheme 6

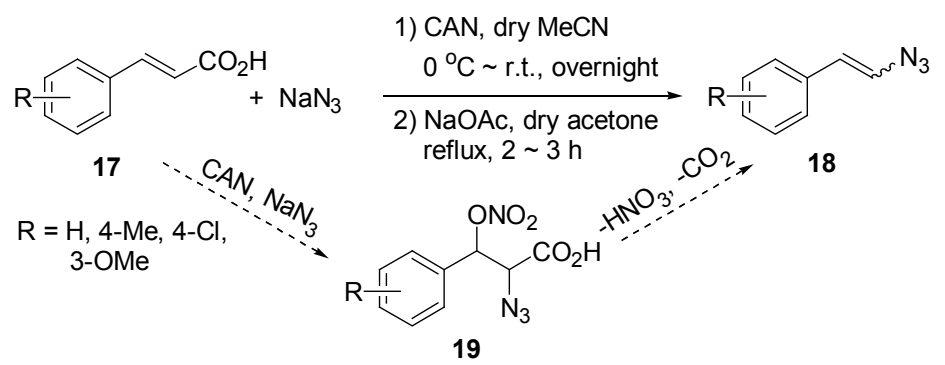

Scheme 7 


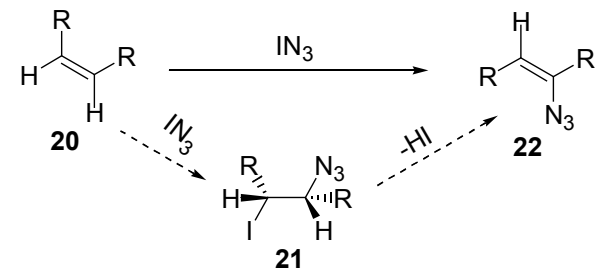

Scheme 8

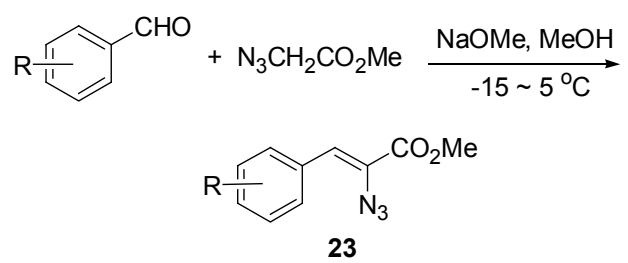

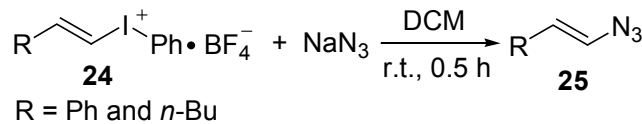

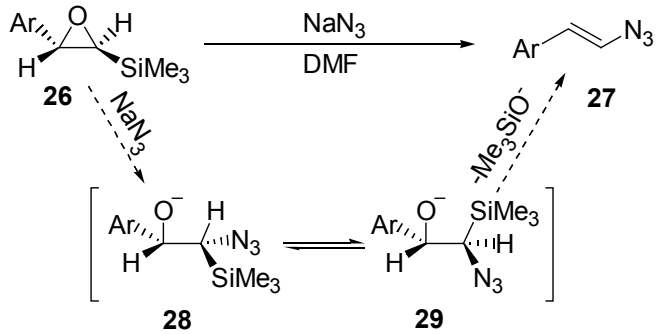

Scheme 9

\section{3 烷基叠氮化合物的合成}

\section{1 卤代烃的亲核取代反应}

烷基或苄基卤代烃与 $\mathrm{NaN}_{3}$ 反应相对较容易, 不需 金属催化甚至就能直接反应得到脂肪或苠基叠氮化合 物(Eq. 5). 这方法在 20 世纪 50 年代就有报道 ${ }^{[18]}$, 至今仍 被广泛使用 ${ }^{[19]}$. 三氟甲磺酸酯也可直接与叠氮钠反应获 得相应叠氮化合物 ${ }^{[20]}$.

$$
\begin{aligned}
& \mathrm{R}-\mathrm{X}+\mathrm{NaN}_{3} \frac{\text { solvent }}{\text { r.t. or heating }} \mathrm{R}-\mathrm{N}_{3} \\
& \mathrm{R}=\text { alkyl, } \mathrm{Bn} ; \mathrm{X}=\mathrm{Cl}, \mathrm{Br}, \mathrm{I}, \mathrm{OTf}
\end{aligned}
$$

Varma 等 ${ }^{[21]}$ 发现, 以水为溶剂, 卤代烃及其类似物 在微波促进条件下即可与叠氮钠发生亲核取代反应得 到相应有机叠氮化合物(Eq. 6).

$$
\begin{aligned}
& \mathrm{R}-\mathrm{X}+\mathrm{NaN}_{3} \underset{\mathrm{MW}, 70 \sim 100 \mathrm{~W}}{\mathrm{H} 2 \mathrm{O}, 30 \mathrm{~min}} \mathrm{R}-\mathrm{N}_{3} \\
& \mathrm{R}=\text { alkyl, } \mathrm{Bn} ; \mathrm{X}=\mathrm{Cl}, \mathrm{Br} \text {, I, OTf }
\end{aligned}
$$

此反应以水为溶剂, 且不需相转移催化剂, 条件温
和、反应高效、绿色环保，而对底物中的酯基、羧基、 羰基、羟基等官能团均具有很好的兼容性，对于多卤化 物亦可进行多位点的叠氮化反应.

2011 年, Zeng 等 ${ }^{[22]}$ 发现聚乙二醇 400 (PEG 400)能 有效加速卤代烃的叠氮化反应. 在此条件下，烷基卤、 茮基卤、酰基卤、烯丙基卤等底物在室温下即能顺利反 应，得到相应产物(Eq. 7).

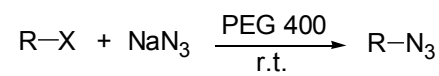

$\mathrm{R}=$ alkyl, cinnamyl, benzoyl, phenacyl, phthalimidyl, glycosyl, etc. $\mathrm{X}=\mathrm{Cl}, \mathrm{Br}, \mathrm{I}$

本方法反应快、条件温和、收率高、普适性好，所 采用的环境友好 PEG 400 能回收循环使用, 且其活性不 减.

\section{2 苄位氢原子直接叠氮化}

Bols 等 ${ }^{[23]}$ 发现，茮基醚 30 可与叠氮化碘能直接发 生反应，生成茮基叠氮 31 , 反应的选择性很好，只发生 在与氧相连的苠位, 其它位置的氢原子则不受影响(Eq. $8)$.

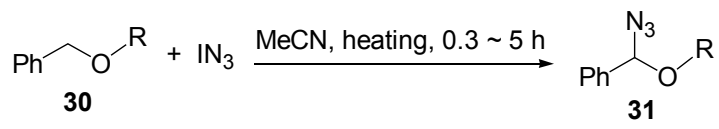

作者认为该反应是自由基机理，叠氮化碘先均裂出 来叠氮自由基，该自由基接着捕获苠位氢原子即产生苠 基自由基，后者再被叠氮化碘或碘分子氧化为苠正离子 并与叠氮离子结合即得产物.

\section{$3.3 \alpha, \beta$-不饱和醛酮与 $\mathrm{NaN}_{3}$ 共轭加成}

Miller 等 ${ }^{[24]}$ 发现，在 1,8-二氮杂环[5,4,0]-7-十一烯 (DBU)催化下， $\alpha, \beta$-不饱和羰基化合物 32 能和三甲基硅 基叠氮( $\left(\mathrm{TMSN}_{3}\right)$ 发生共轭加成, 得到 $\beta$-叠氮基羰基化合 物 33 (Eq. 9).

$$
\mathrm{R}_{32} \stackrel{\mathrm{HOAc}, \mathrm{DBU}(20 \%)}{\text { r.t., } 20 \mathrm{~h}}
$$

33

该方法在室温下即可进行，操作方便，也可以直接 应用于固相合成技术中.

\section{4 伯胺直接叠氮化}

Chiara 等 ${ }^{[25]}$ 报道了有胺直接叠氮化的方法，他们以 全氟丁黄酰基叠氮 34 为叠氮化试剂, 以五水硫酸铜为 催化剂方便的获得了相应有机叠氮化合物(Eq. 10). 


$$
\begin{aligned}
& \mathrm{R}-\mathrm{NH}_{2}+\mathrm{CF}_{3}\left(\mathrm{CF}_{2}\right)_{3} \mathrm{SO}_{2} \mathrm{~N}_{3} \underset{34}{\stackrel{\mathrm{CuSO}_{4} \cdot 5 \mathrm{H}_{2} \mathrm{O}}{\mathrm{Et} \mathrm{O}_{2} / \mathrm{MeOH} / \mathrm{H}_{2} \mathrm{O}}} \mathrm{R}-\mathrm{N}_{3} \\
& \mathrm{R}=\text { aryl or alkyl }
\end{aligned}
$$

本方法所用叠氮化试剂稳定、廉价，反应条件温和， 操作简便, 同时适用于芳胺及脂肪胺. 通过简单萃取操 作即可获得纯化合物, 无需柱层析处理. 若叠氮化完成 后向体系中加入末端炔及还原剂抗坏血酸钠, 则可通过 “一锅法” 区域选择性的获得 1,4-二取代 1,2,3-三氮唑类 化合物.

Katritzky 等 ${ }^{[26]}$ 使用苯并 1,2,3-三唑-1-磺酰基叠氮 35 作为叠氮化试剂, 伯胺在五水硫酸铜催化下也可顺利转 变成相应叠氮化合物, 该方法同时适用于芳基及烷基胺 类化合物(Eq. 11).

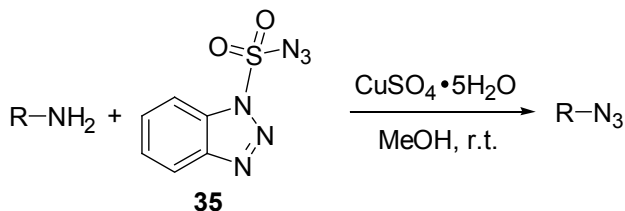

$\mathrm{R}=$ aryl, heteroary, alkyl

本合成方法操作方便、反应高效，对底物中的羧基、 酰基均有很好的兼容性.

\section{5 醇直接叠氮化}

Hajipour 等 ${ }^{[27]}$ 在 $N$-甲基吡咯烷酮类离子液 36 中研 究了叠氮化合物的合成, 在该体系中醇与叠氮化钠可顺 利的反应转变为相应有机叠氮化合物, 离子液同时充当 催化剂及溶剂双重作用(Eq. 12).

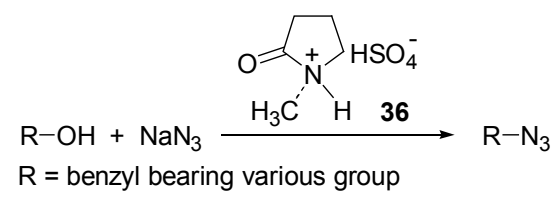

本方法选择性的作用于苠醇及烯丙基醇，而对其它 类型的醇类无对应产物生成，芳环上卤原子不参与反 应, 产品易于分离, 所用的离子液可以循环使用, 符合 绿色化学理念.

手性仲醇或叔醇 37 在二苯基苯氧基磷 $\mathbf{3 8}$ 及三甲基 硅基甲基叠氮化物 39 的作用下可与三甲基硅叠氮 40 直 接反应，选择性的得到相应手性烷基叠氮 41, 此反应在 室温及中性条件下即可顺利进行, 得到构型完全翻转的 产物, 其 $e e$ 值高于 $99 \%$ (Eq. 13) ${ }^{[28]}$.

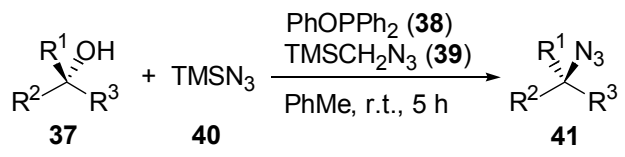

该反应机理如 Scheme 10 所示，二苯基苯氧基磷
(38)与三甲基硅基甲基叠氮化物 39 首先反应生成相应 磷亚胺 42, 后者富电的氮原子接着进攻三甲基硅叠氮的 硅原子，生成中间体 43，该中间体显正电性的磷中心接 受醇的亲核进攻得到中间体 44 , 叠氮负离子最后与 44 发生双分子亲核取代反应即得到构型翻转产物 41 (Scheme 10).

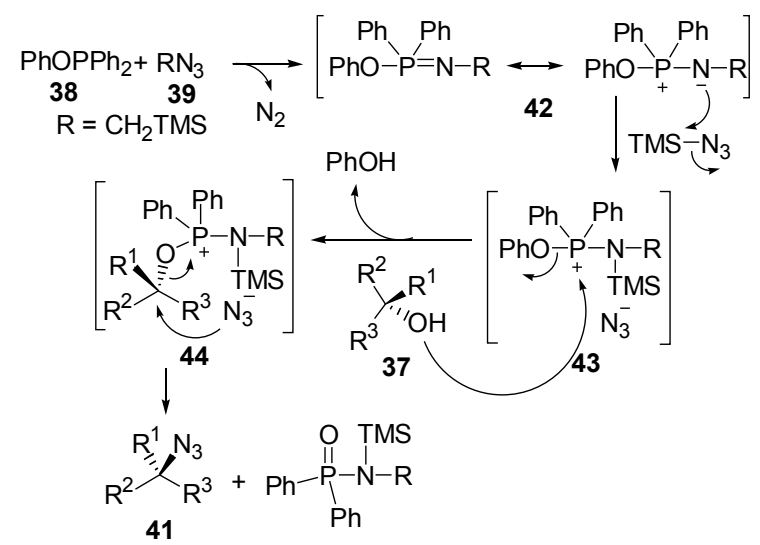

Scheme 10

\section{4 酰基叠氮的合成}

酰基叠氮可用于制备酰胺，合成杂环类化合物，还 可经 Curtius 重排形成异氧酸酯活泼中间体，常用于合 成胺、氨基甲酸酯和脲等. 酰基叠氮主要来源于羰基化 合物，常通有以下方法.

\section{1 以酰肼为原料}

酰肼的重氮化反应是获取酰基叠氮的一条路线，4溴吡啶-2,6-二甲酰腙 44 在酸性条件下经亚硝酸钠处理 即得到相应 4-溴吡啶-2,6-二甲酰基叠氮 45, 后者可用于 胸腺 2A 受体潜在抑制剂的制备(Eq. 14) ${ }^{[29]}$.<smiles>NNC(=O)c1cc(Br)cc(C(N)=O)n1</smiles><smiles>O=[N+]([O-])[O-]</smiles><smiles>NC(=O)c1cc(Br)cc(C(N)=O)n1</smiles>

该方法所用底物酰肼可由相应酰胺或羧酸酯与肼 反应原位生成 ${ }^{[30]}$.

\section{2 以酰胺类化合物为原料}

酰胺除了通过中间体酰肼氧化进行叠氮化外，在一 定条件下还可直接叠氮化得到目标产物. 2010 年 Kong 等 ${ }^{[31]}$ 发现，酰基苯并三唑 46 与叠氮化钠在三氟甲磺酸 锌催化下反应可获得酰基叠氮 47, 以乙酸乙酯和水作为 混合溶剂，反应在室温下即可顺利进行，并获得优良收 率(Eq. 15). 


$$
\text { 等 }
$$

2011 年 Wang 等 ${ }^{[32]}$ 对该体系进行了改进, 他们使用 六水氯化铁作为催化, 反应底物的范围得以扩展，烷 基、芳基、杂环芳基、芳乙烯基等类型反应物均可顺利 进行叠氮化反应，得到相应产物(Eq. 16).

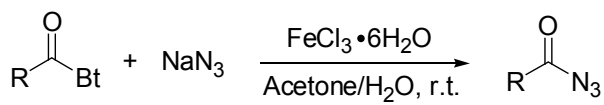

$\mathrm{R}=$ alkyl, aryl, heteroaryl, arylvinyl, etc.

本反应在室温下可顺利进行, 底物转化彻底, 反应 副产物及催化剂通过简单的萃取及洗涤即可除去, 产品 不需柱层析纯化处理，本方法适合放大制备.

\section{3 以羧酸酯为原料}

羧酸酯除了可通过酰胺及酰肼中间体转化为目标 产物外, 还可直接叠氮化得到酰基叠氮化合物. Rawal 等 ${ }^{[33}$ 利用羧酸酯 48 和叠氮二乙基铝(DEAA) 49 反应直 接获得了酰基叠氮(Eq. 17).

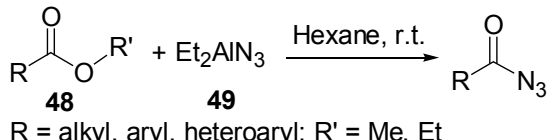

本体系中甲基酯活性要远高于乙基酯, 而缺电的底 物则反应效果差. 后来 $\mathrm{Gao}$ 等 ${ }^{[34]}$ 对该反应机理进行了详 细研究, 他们认为反应中羧酸酯与 DEAA 通过羰基氧与 铝发生配位, 这一方面使 DEAA 由三聚体形式解离成单 体，另一方面酯羰基得以活化，从而促使反应进行.

\section{4 以酰氯为原料}

酰氯除了通过转变成其他羧酸衍生物来合成酰基 叠氮外, 还可与叠氮化钠或叠氮酸直接反应制备相应的 酰基叠氮化合物. Padwa 等 ${ }^{[35]}$ 使用 $\alpha$-氯酰基呋喃类化合 物 50 与叠氮化钠反应获得了相应呋喃类酰基叠氮 51 , 并由其合成了多种 2-位氨基化的呋喃类化合物, 所用的 酰氯可由相应羧酸与二氯亚砜反应原位生成, 不经分离 直接使用(Eq. 18).

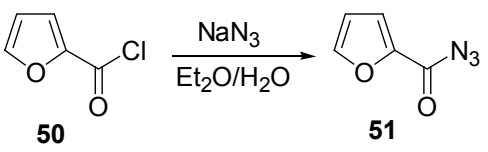

Shirodkar 等 ${ }^{[36]}$ 研究了以水为溶剂的酰氯叠氮化, 他们发现四氢呋喃(THF)能显著促进该反应的进行, 以
含 $5 \% \mathrm{THF}$ 的水溶液作为溶剂, 反应能顺利进行(Eq. 19).

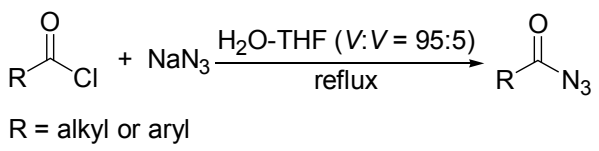

在此条件下，烷基甾即其类似物也能顺利反应得到 相应烷基叠氮化合物。

最近, Liu 等 ${ }^{[37]}$ 利用二茂铁甲酰氯 52 合成了二茂铁 甲酰基叠氮 53 , 所用二茂铁甲酰氯可由相应二茂铁甲酸 与五氯化磷反应获得(Eq. 20).

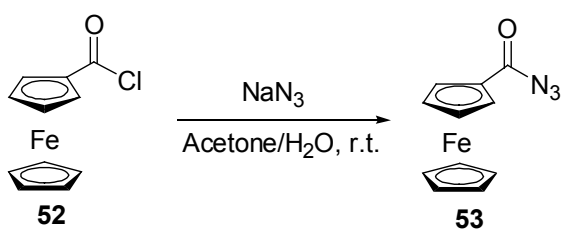

制备的二茂铁甲酰基叠氮与芳胺在 Curtius 重排的 加热条件下与胺进一步反应得到含脲结构的一系列二 茂铁衍生物，后者对 HIV-1 蛋白酶具有一定的抑制活性.

\section{5 以羧酸为原料}

羧酸除了可通过其衍生物转化为酰基叠氮外, 在活 化剂存在时还可与叠氮化合物直接反应获得酰基叠氮 化合物. Gumaste 等 ${ }^{[38]}$ 用三光气作活化剂, 羧基与叠氮 钠反应合成酰基叠氮化合物，无 Curtius 重排，产率高 达 $95 \%$, 其缺点是三光气昂贵且毒性大. Bandgar 等 ${ }^{[39]}$ 报道了合成酰基叠氮的通用方法，在 $N$-甲基吗啉(54)、 三聚氧氯 $(55)$ 及叠氮钠存在下，芳香及脂肪类羧酸可通 过中间体 56 顺利的转化为相应酰基叠氮化合物(Scheme $11)$.

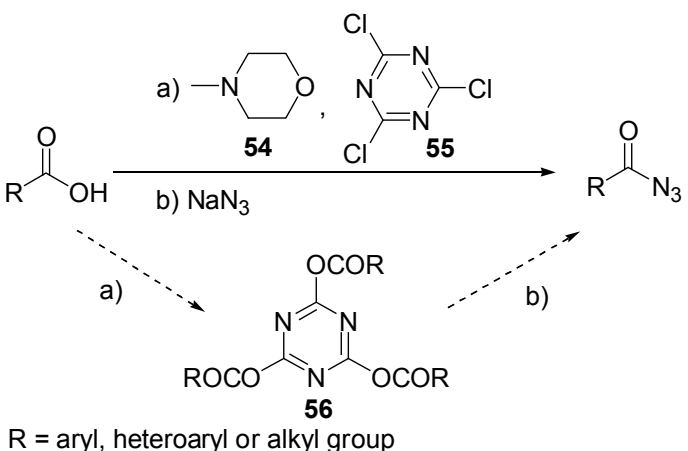

\section{Scheme 11}

Kangani 等 ${ }^{[40]}$ 发现，羧酸在双(2-甲氧基乙基)氨基三 氟化硫(Deoxo-Fluor)作用下与叠氮钠进行一步反应即可 获得相应酰基叠氮(Scheme 12). 


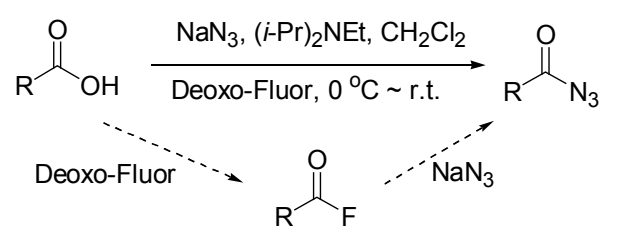

Scheme 12

此反应条件温和、高效，收率高达 $95 \%$, 反应时叠 氮化钠需配成 $0.5 \mathrm{~mol} / \mathrm{L}$ 的 DMSO 溶液, Deoxo-Fluor 需 要最后滴加. 研究表明, Deoxo-Fluor 在反应中作为氟化 试剂, 将羧酸转变成反应中间体酰基氟化物, 后者再与 叠氮化钠发生亲核取代反应得到目标产物.

最近, Nowrouzi 等 ${ }^{[4]}$ 发现了 “叠氮化钠/氯化二苯基 磷/碘” 叠氮化体系, 以二氯甲烷为溶剂, 羧酸在室温下 即可顺利的转变为酰基叠氮化合物(Scheme 13).

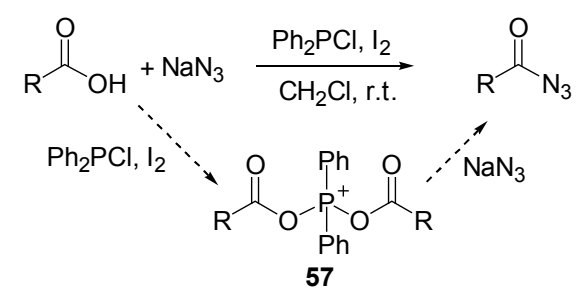

$\begin{aligned} \mathrm{R}= & \mathrm{Ph}, 2 \text { or } 4-\mathrm{NO}_{2} \mathrm{C}_{6} \mathrm{H}_{4}, 4-\mathrm{MeOC}_{6} \mathrm{H}_{4}, 4-\mathrm{ClC}_{6} \mathrm{H}_{4} \text {, } \\ & \text { 2- } \mathrm{BrC}_{6} \mathrm{H}_{4}, \mathrm{Bn}, \mathrm{Et} \text {, etc. }\end{aligned}$

Scheme 13

在反应中羧酸首先与氯化二苯基磷/碘 $\left(\mathrm{Ph}_{2} \mathrm{PCl} / \mathrm{I}_{2}\right)$ 原 位生成酰氧基磷正离子中间体 $\mathbf{5 7}$, 后者羰基接受叠氮离 子进攻即得到目标产物.

\section{6 以醛为原料}

醛在戴斯-马丁试剂 58 作用下, 醛可与叠氮化钠直 接反应得到相应有机叠氮化合物(Eq. 21). 该方法具有 速度快、操作简便、条件温和、收率高等优点, 反应一 步操作即可完成, 不发生 Curtius 重排 ${ }^{[42]}$.

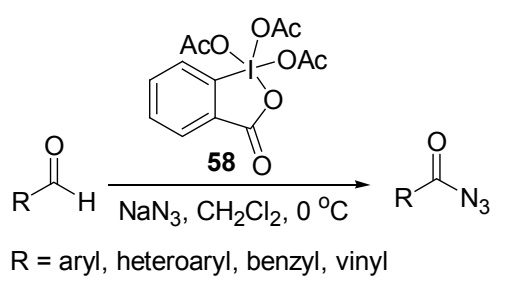

在碘叠氮 59 的作用下, 醛也能顺利转变为相应叠 氮化合物, 该方法适用于脂肪醛及芳香酫 ${ }^{[43]}$, 反应要求 在 $0 \sim 25{ }^{\circ} \mathrm{C}$ 下进行, 若温度过高则易发生 Curtius 重排 生成异氧酸酯 60 , 后者可与叠氮钠进一步反应生成氨酰 基叠氮 61 (Scheme 14).

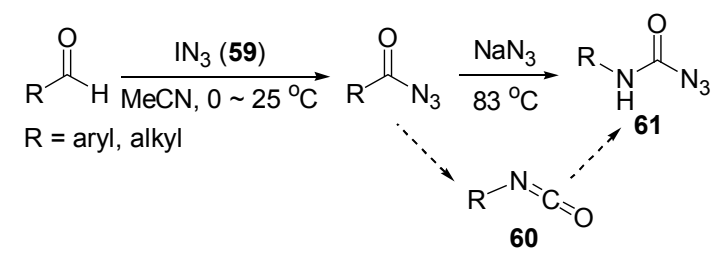

Scheme 14

若向体系中加入自由基捕获剂则无产物生成，表明 该碘叠氮的叠氮化反应属自由基机理，反应过程如 Scheme 5 所示, 碘叠氮 59 弱的碘一叠氮键先发生均裂 生成叠氮自由基 62 及碘自由基 63 , 后者接着掘取醛基 氢原子, 所得到的羰基碳自由基 64 接着与碘叠氮反应 生成相应酰基叠氮 65 和另一份碘自由基, 整个反应只 有碘化氢一种副产物(Scheme 15).

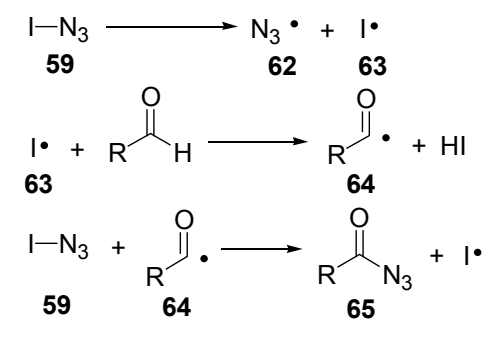

Scheme 15

\section{5 结论与展望}

近年来，随着有机叠氮化合物应用领域的扩大，该 类化合物的合成研究迅速，其合成方法正不断地得到改 进和创新，但在众多尤其是生物学领域的应用仍存在很 多问题及困难，诸如苛刻反应条件及体系残留造成的细 胞损害、试剂放大制备的潜在危险及产品长期储存的弊 端等. 而寻求生物兼容的温和条件、探索低副产物的合 成方法及设计更加多样化的产品结构必将是未来相关 工作者的主要关注方向.

\section{References}

[1] Brase, S.; Gil, C.; Knepper, K.; Zimmermann, V. Angew. Chem., Int. Ed. 2005, 44, 5188.

[2] (a) Sletten, E. M.; Bertozzi, C. R. Angew. Chem., Int. Ed. 2009, 48, 6974.

(b) Jewett, J. C.; Bertozzi, C. R. Chem. Soc. Rev. 2010, 39, 1272.

[3] (a) Iha, R. K.; Wooley, K. L.; Nystrom, A. M.; Burke, D. J.; Kade, M. J.; Hawker, C. J. Chem. Rev. 2009, 109, 5620.

(b) Golas, P. L.; Matyjaszewski, K. Chem. Soc. Rev. 2010, 39, 1338.

(c) Bohn, M. A.; Hammerl, A.; Harris, K.; Klapotke, T. M. Cent. Eur. J. Energ. Mater. 2005, 2, 3.

(d) Niu, X.; Zhang, J.; Wang, Y.; Chen. T.; Zhang, S.; Zhang, T.; Zhou, Z.; Yang, L. Acta Chim. Sinica 2011, 69, 610 (in Chinese). (牛晓庆, 张建国, 王颖, 陈陶平, 张绍文, 张同来, 周遵宁, 杨 利, 化学学报, 2011, 69, 610.)

[4] Griess, P. Liebigs Ann. Chem. 1866, 137, 39.

[5] (a) Rostovtsev, V. V.; Green, L. G.; Fokin, V. V.; Sharpless, K. B. Angew. Chem., Int. Ed. 2002, 41, 2596. 
(b) Kolb, H. C.; Finn, M. G.; Sharpless, K. B. Angew. Chem., Int. Ed. 2001, 40, 2004.

[6] (a) Maryam, C. H.; Roghayeh, C.; Maryam, B.; Ghaffar, H.; Hamed, T. Chin. J. Chem. 2012, 30, 223.

(b) Stengel, I.; Strassert, C. A.; Plummer, E. A.; Chien, C. H.; Cola, L.; Bäuerle, P. Eur. J. Inorg. Chem. 2012, 1795.

(c) Budin, I.; Devaraj, N. K. J. Am. Chem. Soc. 2012, 134, 751.

(d) Sohn, C. H.; Lee, J. E.; Sweredoski, M. J.; Graham, R. L. J.; Smith, G. T.; Hess, S.; Czerwieniec, G.; Loo, J. A.; Deshaies, R. J.; Beauchamp, J. L. J. Am. Chem. Soc. 2012, 134, 2672.

(e) Wei, J.; Chen, J.; Xu, J.; Cao, L.; Deng, H.; Sheng, W.; Zhang, H.; Cao, W. J. Fluorine Chem. 2012, 133, 146.

[7] Ciocoiu, C.; Nikolic, N.; Nguyen, H.; Thoresen, G. H.; Aasen, A. J.; Hansen, T. V. Eur. J. Med. Chem. 2010, 45, 3047.

[8] Zarei, A.; Hajipour, A. R.; Khazdooz, L.; Aghaei, H. Tetrahedron Lett. 2009, 50, 4443.

[9] Lowe-Ma, C.; Nissan, R.; Wilson, W. J. Org. Chem. 1990, 55, 3755.

[10] Zhu, W.; Ma, D. Chem. Commun. 2004, 888.

[11] Tao, C.; Cui,X.; Li, J.; Liu, A.; Liu, L.; Guo, Q. Tetrahedron Lett. 2007, 48, 3525.

[12] Jin H.; Huang, Z. D.; Kuang, C. X.; Wang, X. K. Chin. Chem. Lett. 2011, 22, 310 .

[13] Nair, V.; George, T. Tetrahedron Lett. 2000, 41, 3199.

[14] Hassner, A.; Levy, L. J. Am. Chem. Soc. 1965, 87, 4203.

[15] Brien, A.; Levesque, F.; Seeberger, P. Chem. Coтmun. 2011, 47, 2688.

[16] Yan, J.; Jin, H.; Chen, Z. J. Chem. Res. 2007, 233.

[17] Chakraborty, T.; Reddy, G. Tetrahedron Lett. 1990, 31, 1335.

[18] Boyer, J.; Hamer, J. J. Am. Chem. Soc. 1955, 77, 951.

[19] (a) Lobez, J.; Swager, T. Angew. Chem., In. Ed. 2010, 49, 95. (b) Fuchs, M.; Goessler, W.; Pilger, C.; Kappe, C. Adv. Synth. Catal. 2010, 352, 323.

[20] Zheng, W.; Hsieh, Y.; Chiu, Y.; Cai, S.; Cheng, C.; Chen, C. J. Mater. Chem. 2009, 19, 8432.

[21] Ju, Y.; Kumar, D.; Varma, R. S. J. Org. Chem. 2006, 71, 6697.

[22] Zeng, H.; Tian, Q.; Shao, H. Green Chem. Lett. Rev. 2011, 4, 281.

[23] Viuf, C.; Bols, M. Angew. Chem., Int. Ed. 2001, 40, 623.
[24] Guerin, D.; Horstmann, T.; Miller, S. Org. Lett. 1999, 1, 1107.

[25] Suarez, J. R.; Trastoy, B.; Perez-Ojeda, M. E.; Marin-Barrios, R.; Chiara, J. L. Adv. Synth. Catal. 2010, 352, 2515.

[26] Katritzky, A. R.; Khatib, M. E.; Bol'shakov, O.; Khelashvili, L.; Steel, P. J. J. Org. Chem. 2010, 75, 6532.

[27] Hajipour, A. R.; Rajaei, A.; Ruoho, A. E. Tetrahedron Lett. 2009, 50,708 .

[28] Mukaiyama, T.; Kuroda, K.; Maruyama, Y.; Hayashi, Y. Chem. Lett. 2008, 37, 1072.

[29] Nettekoven, M. Synlett 2001, 1917.

[30] Ikeda, R.; Iwaki, T.; Iida,T.; Okabayashi, T.; Nishi, E.; Kurosawa, M.; Sakai, N.; Konakahara, T. Eur. J. Med. Chem. 2011, 46, 636.

[31] Kong, L.; Hu, J.; Zhang, L. J. Zhejiang Univ. (Nat. Sci.) 2010, 33, 185 (in Chinese).

(孔黎春, 胡洁玲, 孔龙峰, 浙江师范大学学报(自然科学版), 2010, 33, 185.)

[32] Zhong, Z.; Hu, J.; Wang, X.; Liu, J.; Zhang, L. Synth. Commun. 2011, 41, 2461.

[33] Rawal, V. H.; Zhong, H. M. Tetrahedron Lett. 1994, 35(28), 4947.

[34] Gao, Z.; Zhang, X. Chin. J. Inorg. Chem. 2002, 18, 683 (in Chinese).

(高占先, 张小航, 无机化学学报, 2002, 18, 683.)

[35] Padwa, A.; Crawford, K. R.; Rashatasakhon, P.; Rose, M. J. Org. Chem. 2003, 68, 2609.

[36] Swetha, M.; Ramana, P. V.; Shirodkar, S. G. Org. Prep. Proced. Int. 2011, 43, 348.

[37] Liu, W.; Tang, Y.; Guo, Y.; Sun, B.; Zhu, H.; Xiao, Y.; Dong, D.; Yang, C. Appl. Organomet. Chem. 2012, 26, 189.

[38] Gumaste, V. K.; Bhawal, B. M.; Deshmukh, A. R. A. S. Tetrahedron Lett. 2002, 43, 1345.

[39] Bandgar, B. P.; Pandit, S. S. Tetrahedron Lett. 2002, 43, 3413.

[40] Kangani, C. O.; Day, B. W.; Kelley, D. E. Tetrahedron Lett. 2007, $48,5933$.

[41] Nowrouzi, N.; Jonaghani, M. Z. Chin. Chem. Lett. 2012, 23, 442.

[42] Bose, D. S.; Reddy, A. V. N. Tetrahedron Lett. 2003, 44, 3543.

[43] Marinescu, L.; Thinggaard, J.; Thomsen, I. B.; Bols, M. J. Org. Chem. 2003, 68, 9453.

(Cheng, F.) 